\title{
Expression of OPG, RANKL, and RUNX2 in rabbit periodontium under orthodontic force
}

\author{
B. Li ${ }^{1}$, Y.H. Zhang' ${ }^{2}$, L.X. Wang ${ }^{3}$, X. $\mathrm{Li}^{4}$ and X.D. Zhang ${ }^{5}$
}

${ }^{1}$ Department of Stomatology,

Oriental Hospital of Beijing University of Chinese Medicine, Beijing, China

${ }^{2}$ Department of Stomatology,

The Southern District of Beijing Stomatological Hospital, Beijing, China

${ }^{3}$ Department of Stomatology, Beijing Stomatological Hospital, Beijing, China

${ }^{4}$ Department of Anesthesiology,

The Second Artillery General Hospital of Beijing, Beijing, China

${ }^{5}$ Department of Oral and Basic Medicine,

Hebei Medical University, Shijiazhuang, Hebei, China

Corresponding author: Y.H. Zhang

E-mail: yanhuazhangz@126.com

Genet. Mol. Res. 14 (4): 19382-19388 (2015)

Received August 22, 2015

Accepted October 16, 2015

Published December 29, 2015

DOI http://dx.doi.org/10.4238/2015.December.29.48

ABSTRACT. This study aims to investigate the expression changes of $R A N K L, R U N X 2$, and OPG in rabbit periodontal tissues under orthodontic force and explore its effect on the remodeling of periodontal tissues. A total of 16 specific pathogen-free rabbits were used in this study. The maxillary appliance was worn on the right (experimental) side, and the appliance-free left side was used as the control. Rabbits were sacrificed after 3, 5, 7, and 14 days of treatment. Changes in the expression levels of OPG, RANKL, and RUNX2 in the periodontium were detected using real-time PCR and western blotting methods. The OPG expression levels decreased after 3 to 14 days of treatment, while the expression levels of RANKL and RUNX2 increased after 3 to 14 days. The OPG expression levels decreased while those of RANKL and RUNX2 increased during orthodontic tooth movement, 
which suggested that they play a role in the osteogenesis process and the reconstruction of periodontal tissue.

Key words: Orthodontic force; Periodontium; OPG; RANKL; RUNX2; Western blotting

\section{INTRODUCTION}

Fault micromaxillary deformity refers to irregular teeth, upper and lower dental arch dislocation, abnormal jaw position, or size and other deformities caused by genetic or environmental factors during growth and development. It is a common disease that affects the facial appearance and function, and it causes dental caries, periodontal disease, and temporomandibular joint disease (Indira et al., 2014; Al-Moraissi et al., 2015). Orthodontic treatment is the main treatment method for fault micromaxillary deformity, and it can improve the occluding relationship in order to solve problems associated with aesthetics and function. The orthodontic force acting on the periodontal tissue can activate osteoblasts and osteoclasts, and this leads to the absorption and deposition of the alveolar bone, which are accompanied by cell growth and collagen renewal (Wise and King, 2008; Wise, 2009).

The molecular mechanisms of orthodontic tooth movement are not clear. Studies demonstrated that cementoblasts that were stimulated by mechanical signals affected cementoblast functions (Brezniak and Wasserstein, 2002). Osteoprotegerin (OPG) and receptor activator of nuclear factor-kB ligand $(R A N K L)$ were thought to be widely involved in the growth of bone cells (Rody et al., 2001; Wagner and Fahrleitner-Pammer, 2010). However, whether mechanical stimulation affected the expression of OPG, RANKL, and runt-related transcription factor-2 (RUNX2) remained unclear. Therefore, we explored this question in the current study using an animal model.

\section{MATERIAL AND METHODS}

\section{Experimental animals}

A total of 16 specific pathogen-free (SPF) rabbits (weighing 1.0 to $2.5 \mathrm{~kg}$ ) were obtained from the Animal Experimental Center of Vital River Laboratories (Beijing, China). The rabbits were pre-fed for seven days, and they had free access to food and water while adapting to the environment. Food and water were regularly changed, and good room ventilation and natural lighting were present in housing area. The rabbits were anesthetized with $2 \%$ pentobarbital sodium $(2 \mathrm{~mL} / \mathrm{kg})$ via the ear vein. The maxillary appliance $(0.78 \mathrm{~N}$ force) was worn on the right (experimental) side, and the appliance-free left side was used as a control. Rabbits were sacrificed after $3,5,7$, and 14 days of treatment.

Housing and procedures involving experimental animals were in accordance with the Guide for the Care and Use of Laboratory Animals. All experimental procedures were approved by the Care of Experimental Animals Committee of our hospital.

\section{Real-time PCR}

Total RNA was extracted from periodontal tissue using SunShineBio ${ }^{\text {TM }}$ Total RNA 
Extraction Reagent (SunShineBio Ltd., Hnagzhou, China) according to the manufacturer protocol. Total RNA was reverse transcribed and qRT-PCR was conducted using SYBR Green Supermix (Bio-Rad-172-5264, USA). The PCR primers are listed in Table 1. The amplification conditions were as follows: $94^{\circ} \mathrm{C}$ for $5 \mathrm{~min}$ followed by 40 cycles of $94^{\circ} \mathrm{C}$ for $30 \mathrm{~s}, 56^{\circ} \mathrm{C}$ for $30 \mathrm{~s}$, and $72^{\circ} \mathrm{C}$ for $30 \mathrm{~s}$. For relative quantification, we calculated the $n$-fold differential expression using the $2^{-\Delta \Delta C t}$ method (Ct denotes the threshold PCR amplification cycle at which product is first detected by fluorescence). This method compares the amount of target gene amplification, which is normalized to the GAPDH endogenous reference.

\begin{tabular}{llll}
\multicolumn{2}{l}{ Table 1. Primers used in real-time PCR analyses. } \\
\hline Gene & GenBank accession No. & Primer pair (5'-3') & Length of product (bp) \\
\hline OPG & NM_002546.3 & F: AACCCCAGAGCGAAATAC & 205 \\
RANKL & NM_057149.1 & R: AAGAATGCCTCCTCACAC & 101 \\
RUNX2 & XM_008262992.1 & R: TTAAAAGCCCCAAAGTATG & 172 \\
GAPDH & FM002046 & R: ACTTGGTTTTTCATAACAGCGGA & 141 \\
& & F: CCTCAAGATTGTCAGCAAT & \\
\hline
\end{tabular}

$O P G$ = osteoprotegerin; RANKL = receptor activator of nuclear factor kappa $\mathrm{B}$ ligand; RUNX2 = runt-related transcription factor-2; GAPDH = glyceraldehyde phosphate dehydrogenase; $\mathrm{F}=$ forward; $\mathrm{R}=$ reverse.

\section{Western blotting method}

Total proteins were extracted from periodontal tissue and analyzed with SDS-PAGE electrophoresis. Periodontal tissues were homogenized using RIPA buffer $(10 \mu \mathrm{g} / \mathrm{mL})$ and a protease inhibitor. Total proteins were isolated, and their concentrations were measured using the bicinchoninic acid (BCA) method. Proteins were then electrotransferred to the PVDF membrane, and the membrane containing the proteins was used for immunoblotting with required antibodies. Proteins were blocked with $5 \%$ non-fat milk in TBST buffer for 2 h, and they were then incubated with the primary antibodies (Santa Cruz Biotechnology; sc-8468, sc9073 , and sc-390715) at $4^{\circ} \mathrm{C}$ overnight. Proteins were subsequently incubated with secondary antibodies that were conjugated with horseradish peroxidase for $1 \mathrm{~h}$ at room temperature. $G A P D H$ was used as an internal control. The images were analyzed using the Quantity One (version 4.62) software.

\section{Statistical analysis}

Statistical analysis was performed using the Statistical Package for the Social Science (SPSS, version 18.0) program. The data are reported as means \pm SD, and differences between experimental groups were analyzed using Student $t$-tests. $\mathrm{P}<0.05$ was considered to be significant.

\section{RESULTS}

\section{Real-time PCR results}

Real-time PCR results are shown in Figure 1. The results showed that the OPG expression 
levels were $0.83 \pm 0.06,0.62 \pm 0.04,0.40 \pm 0.06$, and $0.36 \pm 0.07$ after treatment for $3,5,7$, and 14 days, respectively, and they decreased significantly compared to the control group $(P<0.05)$. The RANKL expression levels were $1.52 \pm 0.09,1.41 \pm 0.03,1.38 \pm 0.06$, and $1.26 \pm 0.07$ after treatment for $3,5,7$, and 14 days, respectively, and they increased significantly compared to the control group $(\mathrm{P}<0.05)$. The RUNX2 expression levels were $2.21 \pm 0.09,1.96 \pm 0.03,1.82 \pm$ 0.06 , and $1.88 \pm 0.07$ after treatment for $3,5,7$, and 14 days, respectively, and they increased significantly compared to the control group $(P<0.05)$.
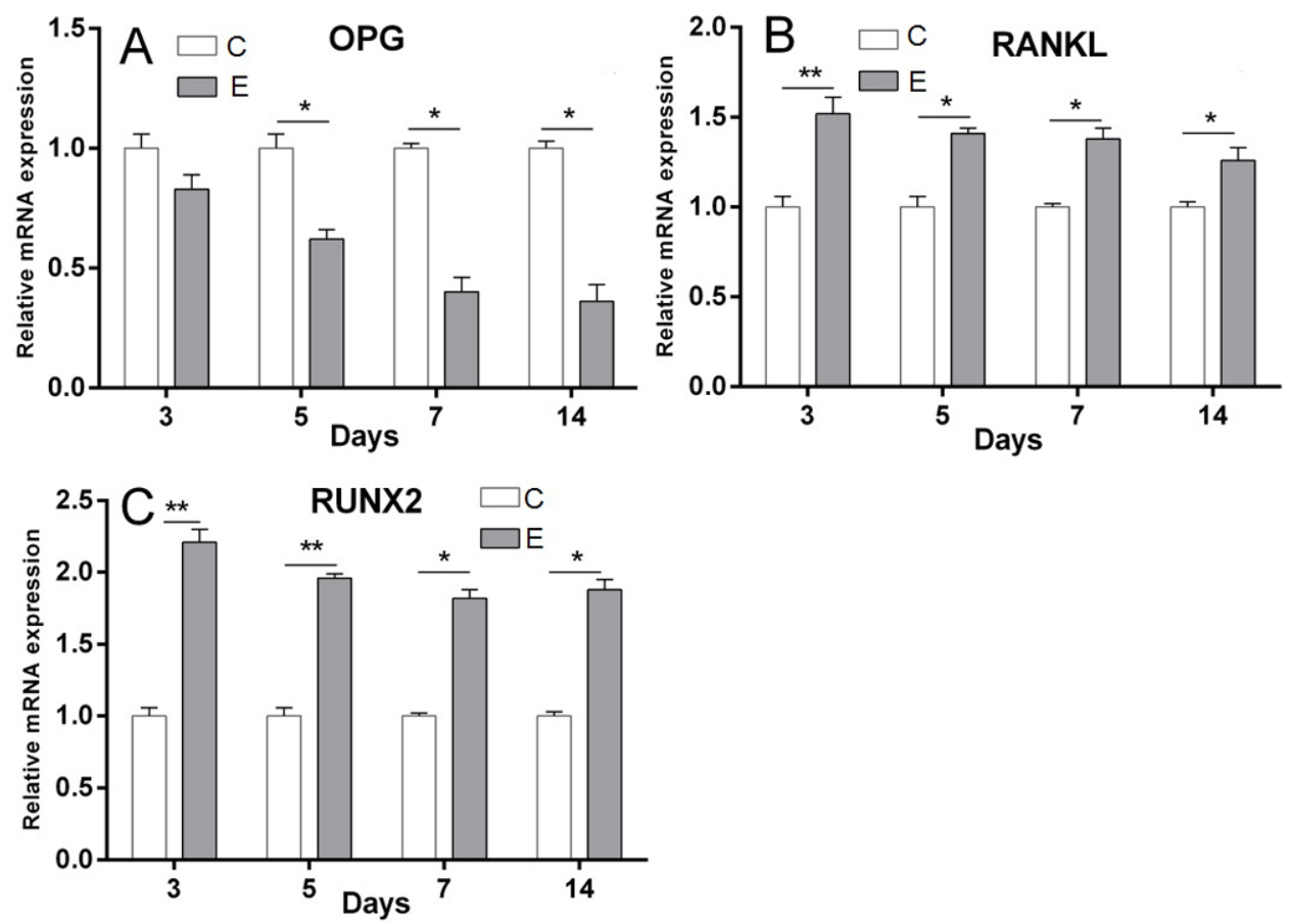

Figure 1. Real-time PCR results. A. Relative expression level of OPG. B. Relative expression level of RANKL. C. Relative expression level of $R U N X 2 ;{ }^{*} \mathrm{P}<0.05 ;{ }^{* *} \mathrm{P}<0.01$. $\mathrm{C}=$ control; $\mathrm{E}=$ experimental group.

\section{Western blotting results}

Western blotting results are shown in Figure 2. The results were similar to the RT-PCR results. The results indicated that the OPG expression levels decreased and those of RANKL and RUNX2 increased significantly compared to the control group after treatment $(P<0.05)$.

\section{DISCUSSION}

Fault micromaxillary deformity is caused by genetic or environmental factors during growth and development. It not only affects facial appearance and function, but it also causes dental caries and periodontal disease (Rody et al., 2001; Indira et al., 2014). 



Figure 2. Western blotting results.

Orthodontic treatment can improve these symptoms. Mechanical force applied to periodontal tissues induced a cellular response, and it resulted in the degradation of collagen and bone tissue absorption on the stressed side. Periodontal ligament cells on the stressed side grew, and bone tissue precipitation occurred, causing bone remodeling and tooth movement (Iliadi et al., 2015; Krishnan et al., 2015).

Orthodontic treatment caused an inflammatory reaction in periodontal tissues under the action of the external force, and orthodontic force was transferred to the periodontal membrane, which induced periodontal tissue remodeling (Thirunavukkarasu et al., 2000; Hendesi et al., 2015). This process required osteoclasts and cementoblasts. Cementoblasts participated in the root repair process and in the regulation of the differentiation and absorption functions of osteoclasts, and they also affected root absorption (Boabaid et al., 2004; Marchesan et al., 2013; Yang et al., 2015). In recent years, the OPG and RANKL system was found to be an important signal pathway in the differentiation of osteoclasts, which was widely involved in bone cell growth. The RANKL receptor is located on the cell membrane of osteoclasts. OPG belongs to the superfamily of TNF receptors, and its main function is to inhibit the differentiation of osteoclasts and to induce apoptosis. However, OPG is a false receptor of RANKL. Osteoblasts expressed RANKL, which can promote the differentiation of bone cells and bone resorption after the gene product combines with RANK. OPG secreted by osteoblasts can competitively combine with RANKL to inhibit the binding between RANK and RANKL (Boyce and Xing, 2007; Tokuyama and Tanaka, 2010; Shimamura et 
al., 2014). In this study, we found that $O P G$ expression was downregulated by orthodontic force, while RANKL expression was upregulated. Therefore, the expression ratio of OPG and RANKL can reflect the early response of cementum cells to mechanical signals. The fibroblast group is a cell group in the periodontal tissues with differentiation potential, and the group can successfully differentiate osteoblasts based on the expression of the phenotype and functional osteoblast proteins that are subjected to mechanical forces. Moreover, osteoblast-specific transcription factors play a central regulatory role in the process. RUNX2 is a specific transcription factor that can activate bone marrow mesenchymal cells for differentiation into osteoblasts. Furthermore, it can regulate the maturation of osteoblasts and OPG expression, and it also plays a regulatory role in bone formation and bone resorption (Baek et al., 2014; Nagatake et al., 2015). In this study we found that mechanical stimulation can upregulate the expression of $R U N X 2$, which may be caused by decreased OPG expression.

\section{Conflicts of interest}

The authors declare no conflict of interest.

\section{REFERENCES}

Al-Moraissi EA and Ellis E 3rd (2015). Surgical treatment of adult mandibular condylar fractures provides better outcomes than closed treatment: a systematic review and meta-analysis. J. Oral Maxillofac. Surg. 73: 482-493.

Baek JE, Choi JY and Kim JE (2014). Skeletal analysis and differential gene expression in Runx2/Osterix double heterozygous embryos. Biochem. Biophys. Res. Commun. 451: 442-448.

Boabaid F, Berry JE, Koh AJ, Somerman MJ, et al. (2004). The role of parathyroid hormone-related protein in the regulation of osteoclastogenesis by cementoblasts. J. Periodontol. 75: 1247-1254.

Boyce BF and Xing L (2007). Biology of RANK, RANKL, and osteoprotegerin. Arthritis Res. Ther. 9: S1.

Brezniak N and Wasserstein A (2002). Orthodontically induced inflammatory root resorption. Part I: the basic science aspects. Angle Orthod. 72: 175-179.

Hendesi H, Barbe MF, Safadi FF, Monroy MA, et al. (2015). Integrin mediated adhesion of osteoblasts to connective tissue growth factor (CTGF/CCN2) induces cytoskeleton reorganization and cell differentiation. PLoS One 10: e0115325.

Iliadi A, Kloukos D, Gkantidis N, Katsaros C, et al. (2015). Failure of fixed orthodontic retainers: a systematic review. J. Dent. 43: 876-896.

Indira MD, Singh Dhull K, Nandlal B, Praveen Kumar PS, et al. (2014). Biological restoration in pediatric dentistry: a brief insight. Int. J. Clin. Pediatr. Dent. 7: 197-201.

Krishnan S, Pandian S and Kumar SA (2015). Effect of bisphosphonates on orthodontic tooth movement-an update. J. Clin. Diagn. Res. 9: ZE01-ZE05.

Marchesan JT, Gerow EA, Schaff R, TautAD, et al. (2013). Porphyromonas gingivalis oral infection exacerbates the development and severity of collagen-induced arthritis. Arthritis Res. Ther. 15: R186.

Nagatake T, Fukuyama S, Sato S, Okura H, et al. (2015). Central role of core binding factor $\beta 2$ in mucosa-associated lymphoid tissue organogenesis in mouse. PLoS One 10: e0127460.

Rody WJ Jr, King GJ and Gu G (2001). Osteoclast recruitment to sites of compression in orthodontic tooth movement. Am. J. Orthod. Dentofacial Orthop. 120: 477-489.

Shimamura M, Nakagami H, Osako MK, Kurinami $\mathrm{H}$, et al. (2014). OPG/RANKL/RANK axis is a critical inflammatory signaling system in ischemic brain in mice. Proc. Natl. Acad. Sci. U. S. A. 111: 8191-8196.

Thirunavukkarasu K, Halladay DL, Miles RR, Yang X, et al. (2000). The osteoblast-specific transcription factor Cbfa1 contributes to the expression of osteoprotegerin, a potent inhibitor of osteoclast differentiation and function. J. Biol. Chem. 275: 25163-25172.

Tokuyama N and Tanaka S (2010). Cytokines in bone diseases. Genetic disorders of RANKL-RANK-OPG system. Clin. Calcium 20: 1532-1538.

Wagner D and Fahrleitner-Pammer A (2010). Levels of osteoprotegerin (OPG) and receptor activator for nuclear factor kappa B ligand (RANKL) in serum: are they of any help? Wien. Med. Wochenschr. 160: 452-457. 
Wise GE (2009). Cellular and molecular basis of tooth eruption. Orthod. Craniofac. Res. 12: 67-73.

Wise GE and King GJ (2008). Mechanisms of tooth eruption and orthodontic tooth movement. J. Dent. Res. 87: 414-434.

Yang X, Wang Y, Han X, Shu R, et al. (2015). Effects of TGF- $\beta 1$ on OPG/RANKL expression of cementoblasts and osteoblasts are similar without stress but different with mechanical compressive stress. Scientific World J. 2015: 718180.

Yasuda H (2013). RANKL, a necessary chance for clinical application to osteoporosis and cancer-related bone diseases. World J. Orthop. 4: 207-217. 